\title{
Calculations of parameters and mathematical model of rotary air motor
}

\author{
Lukáš Dvořák ${ }^{1, *}$, Kamil Fojtášek ${ }^{2}$, Vojtěch Řeháček ${ }^{3}$ \\ 1,2,3 VŠB-Technical University of Ostrava, Faculty of Mechanical Engineering, Department of Hydrodynamics and Hydraulic Equipment, \\ 17. listopadu 15/2172, Ostrava 70833, Czech Republic
}

\begin{abstract}
The article describes calculations of the parameters of rotary air motors, focuses on the mathematical model of air motor using Matlab Simulink-Simscape and concludes with the comparison of the results of the experiment and a the mathematical model.
\end{abstract}

\section{Introduction}

The rotary pneumatic motors are not used to drive machines so often. It is primarily due to the relatively low efficiency of the pneumatic actuator. Other disadvantages are the speed change when the load changes and the noise. Even so, in some applications they are irreplaceable. In these cases on the other hand the benefits are manifested as: simple operation and maintenance, operational reliability, ability to work in potentially explosive atmospheres, the overload does not demage the motor etc. From the perspective of design the pneumatic motors can be divided into vane, piston and gear types. All types may be included in the group of volumetric motors. Turbine motors are in the separate category.

\section{Calculation of basic parameters of rotary motors}

According to the utilization of air in the working space of motor, the motors can be divided into fullpressure motors and motors with partial expansion types, Fig. 1.
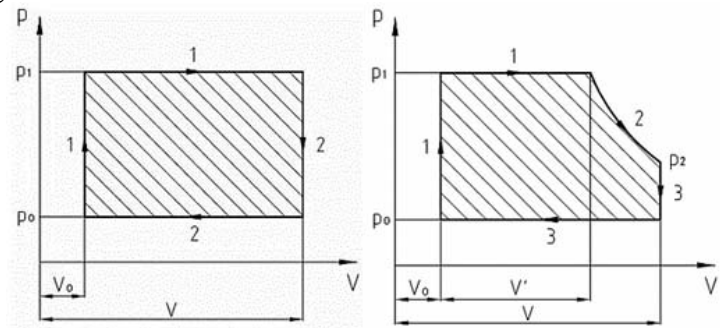

Fig. 1. $\mathrm{p}-\mathrm{V}$ (indicator) diagram of full-pressure motors and motors with partial expansion

For motors with partial expansion the ratio of the volumes $\left.\left(V-V_{0}\right) / V\right)$ is called coefficient of filling $(\delta)$ and it ranges from 0.45 to 0.7 .
Main value for the calculation of the motor parameters is called the indicated pressure and its theoretical value can be calculated for full-pressure motors from equation 1

$$
p_{i t}=\left(p_{1}-p_{0}\right)
$$

and for motors with partial expansion from equation 2 .

$$
p_{i t}=p_{1} \cdot \delta \cdot \frac{n-\delta^{n-1}}{n-1}-p_{0}
$$

A polytropic exponent is in the range of $n=1.25$ to 1.32 depending on the motor type. The problem is that the real value of the indicated pressure will be lower, because at high speeds the working space will not be filled enough, as it is shown in Fig. 2.

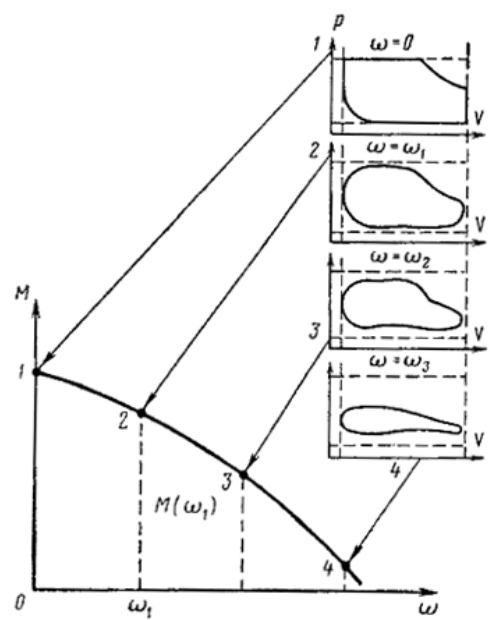

Fig. 2. Influence of speed to filling of work space [3]

The final shape of the indicator diagram and thus the value of mean indicated pressure is also affected by the size and shape of distribution channels and their resistance to airflow. 
The true value of indicated pressure we can obtained from equation 3 ,

$$
p_{i}=p_{i t} \cdot \eta_{p}
$$

where $\eta_{p}$ is filling efficiency of workspace. The efficiency curves for some designs of rotary motors are shown in Fig. 3.

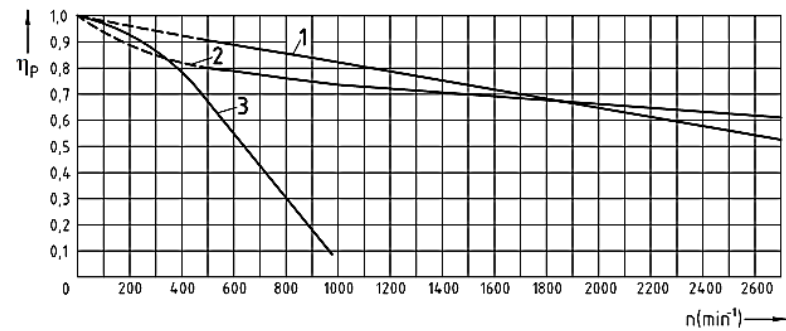

Fig. 3. Filling efficiency 1 - gear motor with spiral gearing, 2 - gear motor with herring-bone gearing 3 - piston motor [3]

The filling efficiency affects the calculation of all the basic parameters throughout the operating speed range. An examples of the torque and power characteristics together with the air consumption are in Fig. 4.

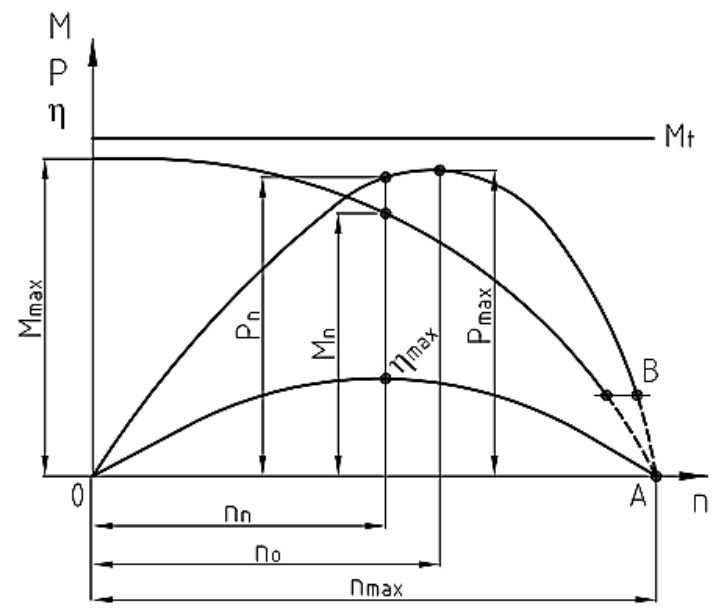

Fig. 4. Characteristics of rotary motors depending on speed

For the calculation of individual parameters we can use the following equations [1]. The theoretical torque is not dependent on the speed. But the calculation of the real torque is influenced by the filling efficiency therefore the characteristic has a downward trend.

$$
M=\frac{1}{2 \pi} \cdot V_{g} \cdot p_{i t} \cdot \eta_{p} \cdot \eta_{m}
$$

In the equation $V_{g}$ is displacement of motor, $p_{i t}$ is theoretical indicated pressure, $\eta_{p}$ is filling efficiency and $\eta_{m}$ is mechanical efficiency, which has value for piston motors with connecting rod mechanism $\eta_{m}=0.85 \div 0.9$ and for motors with a sliding mechanism $\eta_{m}=0.9 \div$ 0.92 .

Power can be calculate from equation 5 .

$$
P_{s}=V_{g} \cdot n \cdot p_{i t} \cdot \eta_{p} \cdot \eta_{m}
$$

From the analysis of experimental measurements of all types of air motors it is evident that the filling efficiency $\eta_{p}$ depending on the speed varies approximately linearly.

This fact can be expressed as follows $\eta_{p}=1-a \cdot n$ where $a$ expresses the inclination of line. After substitution it is therefore possible to calculate the power from the equation 6 .

$$
P_{s}=V_{g} \cdot n \cdot P_{i t} \cdot \eta_{m}-V_{g} \cdot n^{2} \cdot p_{i t} \cdot a \cdot \eta_{m}
$$

The air consumption recalculated to atmospheric normal conditions can be approximately calculated from equation

$$
Q_{n}=V_{g} \cdot n \cdot \frac{p_{1}}{p_{N}} \cdot \delta \cdot \frac{1}{\eta_{Q}}
$$

where $\eta_{Q}$ is flow efficiency, which has a value from about 0.85 to 0.95 , depending on the design and motor wear.

\section{Comparison of measured and calculated characteristics}

The object of our research were mainly small radial piston motors with power up to $500 \mathrm{~W}$. For characteristics measuring test equipment has been compiled, Fig. 5.

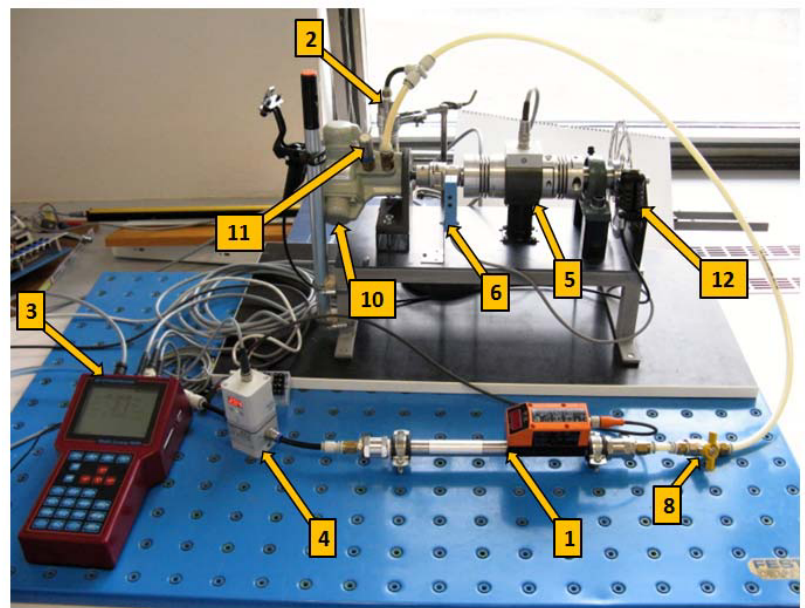

Fig. 5. Test equipment, 1 - flowmeter, 2 - pressure sensor, 3 - recording device, 4 - reduction valve, 5 - torque sensor, 6 - speed sensor, 7 - FRL unit, 8,9 - ball valve, 10 - pneumatic rotary motor, 11 - silencer, 12 - disc brake

The measurement results are the characteristics of the torque, power, air consumption and overall efficiency.

From the measurement results the filling efficiency of radial piston motor was found, Fig. 6. Parameter $a$ expressing the inclination of line in this case has a value $a=0.028$. Further, based on the measurement the mechanical efficiency of the motor was determined. Its value is $\eta_{m}=0.86$. 


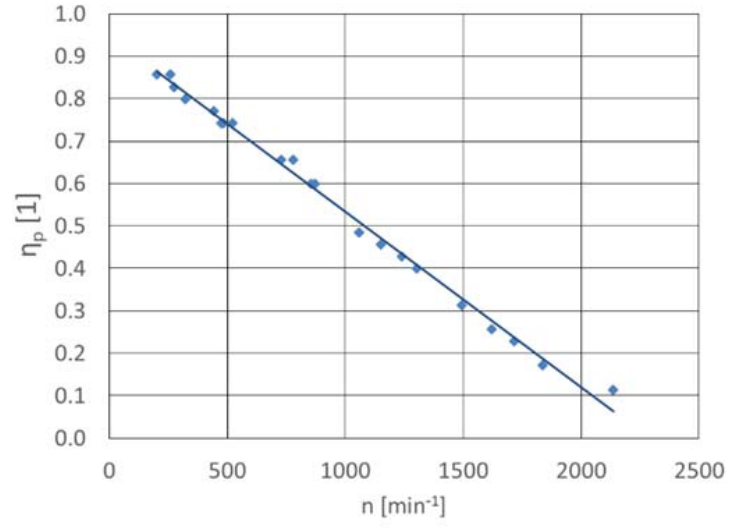

Fig. 6. Filling efficiency of measured motor

When we compare power calculated using equation 6 it well corresponds with the experimental results, Fig. 7. The difference is noticeable at higher speeds, but this area is already outside of the optimum operating range.

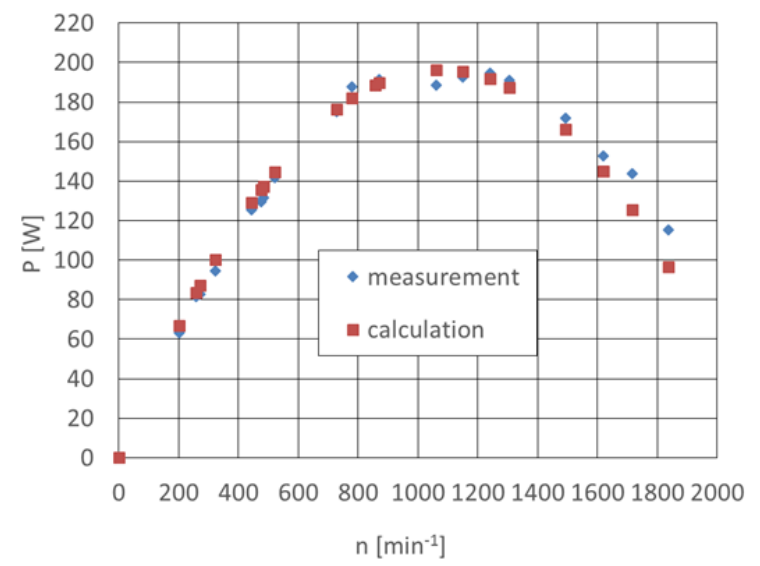

Fig. 7. Power of measured motor

\section{Modelling of a pneumatic system with a rotary motor}

In addition to the measurements we carried out verification of the possibility of mathematical modeling of pneumatic systems with the rotary motor. The MatlabSimulink Simscape was used for modeling. Simscape contains models of the basic pneumatic components including the rotary air motor model "Rotary Pneumatic Piston Chamber". Into this model, it is necessary to specify Displacement (volume per unit angle), Initial angle, Dead volume and Chamber orientation (direction of rotation). Mechanical neither flow efficiency is not included into the mathematical model of the motor. The efficiencies must be added in the calculation externally. But even after these adjustments we have not achieved good results. The torque at zero speed corresponds to the real motor but the torque characteristics depending on the speed do not decrease linearly. Larger differences are then in power characteristics. Maximum calculated power is several times greater than the real power and it is also achieved at higher speeds. With the above model we have not achieved real results.

The manual for Simulink (Help) contains another model, "Pneumatic motor". There is chosen a completely different approach of motor parameters definition. Into the model it is necessary to enter vector of rotational speeds, vector of torque values, vector of volumetric flow rates and pressure differential at which the torque and flow data were measured. By this way the characteristics of the motor can be very precisely defined. The disadvantage is in obtaining these values by measurement. On the other hand, the simulation results are quite accurate. Table 1 shows the values that were entered into the model of rotary motor.

It was also necessary to add the source of constant pressure, which was set at 5 bar, and the models of directional valve and compressed air lines in the model of system, Fig. 8 .

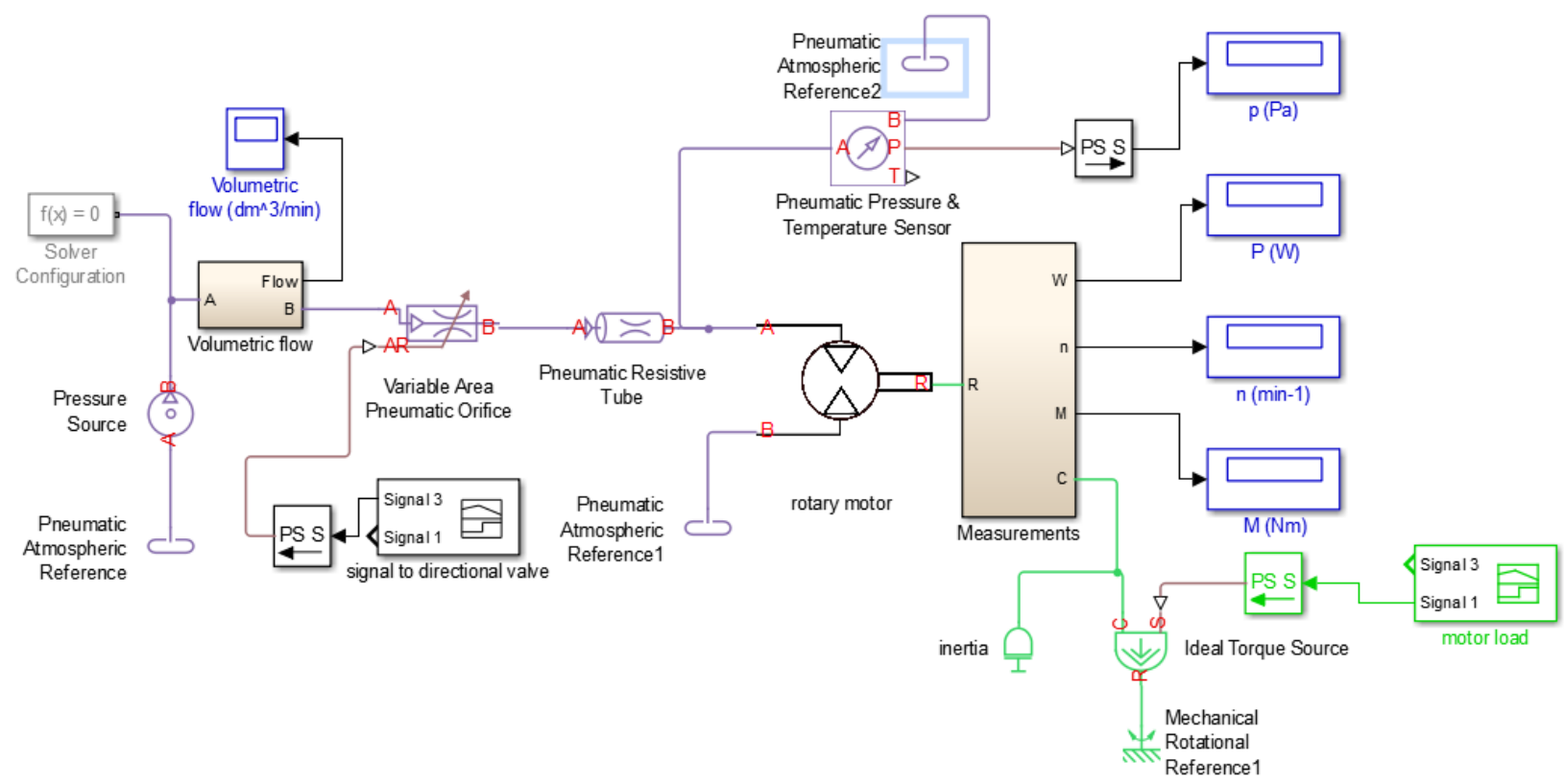

Fig.8. Block diagram of the mathematical model [2] 
The elements for measuring the flow, the pressure, the torque, the speed and the power were also included in the model. The load was simulated in two ways. Firstly, the block "Inertia" was set to $0.001 \mathrm{~kg} \cdot \mathrm{m}^{2}$. This value corresponds to the inertia of the motor and other rotating parts of the test equipment (torque sensor, clutch, shaft and brake disc). It was also necessary to define the load torque. This was realized by the block "Ideal Torque Source". The signal which corresponded with the motor load during the measurement was fed on the input of this block.

Table 1. Values entered into the model

\begin{tabular}{|c|c|c|}
\hline $\begin{array}{c}n \\
{\left[\mathrm{~min}^{-1}\right]}\end{array}$ & $\begin{array}{c}M \\
{[\mathrm{Nm}]}\end{array}$ & $\begin{array}{c}Q \\
{\left[\mathrm{dm}^{3} . \mathrm{min}^{-1}\right] \text { (ANR) }}\end{array}$ \\
\hline 0 & 3.329 & 55 \\
\hline 200 & 3.029 & 119 \\
\hline 400 & 2.729 & 177 \\
\hline 600 & 2.429 & 228 \\
\hline 800 & 2.129 & 273 \\
\hline 1000 & 1.829 & 311 \\
\hline 1200 & 1.529 & 343 \\
\hline 1400 & 1.229 & 369 \\
\hline 1600 & 0.9287 & 388 \\
\hline 1800 & 0.6287 & 401 \\
\hline 2000 & 0.3287 & 407 \\
\hline 2136 & 0.1247 & 435 \\
\hline
\end{tabular}

The measurement and the modeling was performed for several different ways of the load. The first of these was the case when the motor was loaded by a constant torque and the motor accelerated from the zero speed. An example of measured and calculated values is in Fig. 9. In this case, at the time of $1.4 s$ the valve has opened. At the time of approximately $2 s$ the steady speeds was achieved. During the acceleration the torque has risen to $1.9 \mathrm{Nm}$ due to the inertia of the rotating parts, after the acceleration the torque reached the level of $1.1 \mathrm{Nm}$.

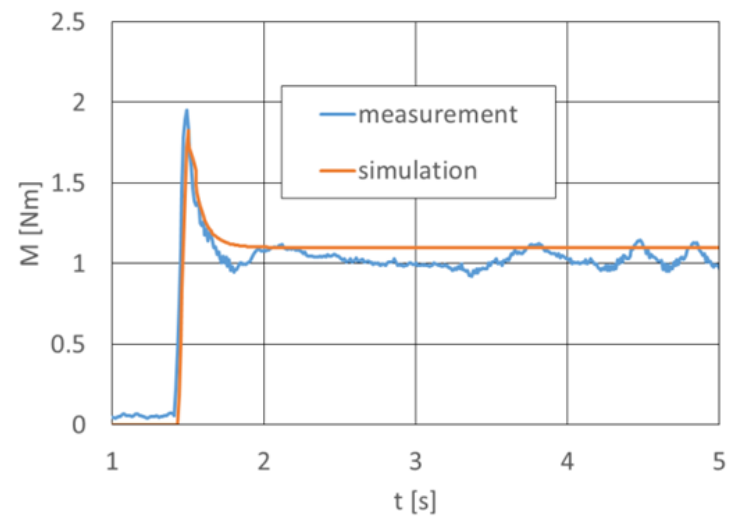

Fig. 9. Torque during motor acceleration
Subsequently, the accuracy of the simulation was validated in the case where during the rotation the load was increased, for example from $0.7 \mathrm{Nm}$ to $0.95 \mathrm{Nm}$. This change in torque has resulted in the decrease of speed from $1245 \mathrm{~min}^{-1}$ to $980 \mathrm{~min}^{-1}$, see Fig. 10. This behavior is typical for the rotary pneumatic motors. Similar results were achieved in all other investigated cases.

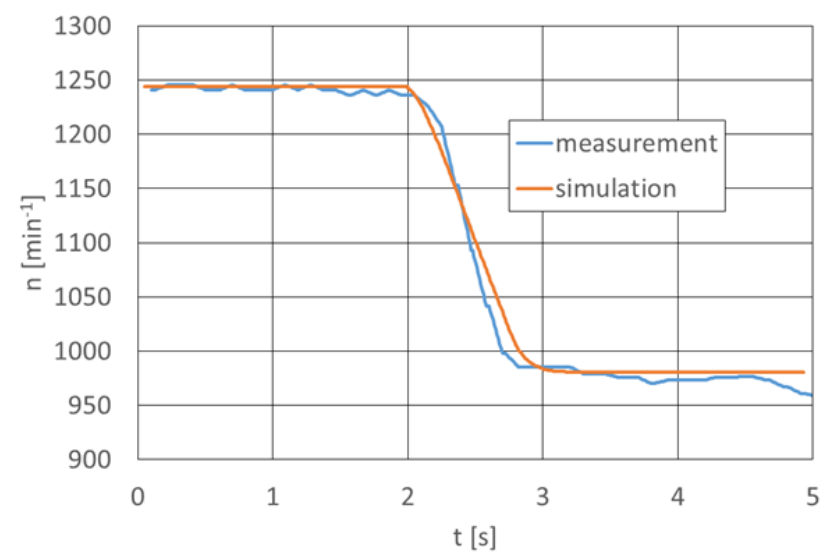

Fig. 10. Speed during load increase

\section{Conclusion}

At the beginning of the article the procedure for calculating the main parameters and characteristics of the rotary air motors was stated. This problem is quite difficult, because the results are influenced by the filling efficiency. The efficiency varies according to the type of engine. For this reason, it is very difficult to predict the characteristics of the motor during its design. Another part is devoted to the possibilities of mathematical modeling of pneumatic system with rotary pneumatic motor. From the calculations it follows that model results, when the basic data are entered, are not very accurate. Relatively accurate results can be achieved with a model in which speed vector and corresponding vectors of torque and air consumption are inserted. The disadvantage of this model is that these values must be detected experimentally.

\section{Acknowledgements}

This work was supported by the project SP2016/70 of VŠB-Technical University of Ostrava.

\section{References}

1. J. Kopáček: Pneumatické mechanismy (1998)

2. V. Reháček: Design of Test Equipment of Pneumatic Rotary Motors: Master Thesis (2016)

3. K.S. Borisenko: Pnevmatičeskije dvigateli gornych mašin (1958) 